\title{
Global Engineering Competency: Assessment Tools and Training Strategies
}

\section{Prof. Brent K. Jesiek, Purdue University, West Lafayette}

Dr. Brent K. Jesiek is an Associate Professor in the Schools of Engineering Education and Electrical and Computer Engineering at Purdue University. He also leads the Global Engineering Education Collaboratory (GEEC) research group, and is the recipient of an NSF CAREER award to study boundary-spanning roles and competencies among early career engineers. He holds a B.S. in Electrical Engineering from Michigan Tech and M.S. and Ph.D. degrees in Science and Technology Studies (STS) from Virginia Tech. Dr. Jesiek draws on expertise from engineering, computing, and the social sciences to advance understanding of geographic, disciplinary, and historical variations in engineering education and practice.

\section{Prof. Natascha Trellinger Buswell, University of California, Irvine}

Natascha Trellinger Buswell is an assistant teaching professor in the department of mechanical and aerospace engineering at the University of California, Irvine. She received her B.S. in aerospace engineering from Syracuse University and her Ph.D. in engineering education from the School of Engineering Education at Purdue University. She is particularly interested in teaching conceptions and methods and graduate level engineering education.

\section{Dr. Qin Zhu, Colorado School of Mines}

Qin Zhu is Research Assistant Professor in the Ethics Across Campus Program and the Division of Humanities, Arts \& Social Sciences at Colorado School of Mines, where he is also helping with managing the Daniels Fund Faculty Fellows Program that provides scholarly and grant support for faculty to explore ways to integrate ethics into their applied science and engineering curricula. Qin is also completing his second PhD degree in the School of Engineering Education at Purdue University. Qin holds a bachelor's degree in Materials Engineering and a PhD in Philosophy (concentration in Ethics of Technology and Engineering) from Dalian University of Technology (DUT) (Dalian, China). Qin has broad teaching and research interests in the ethical, historical-cultural, and policy perspectives of engineering practice and education. His research has drawn on theories, methods, and practices from a wide range of fields including philosophy of technology, engineering ethics, engineering education, and Confucian ethics. His work has appeared in peer-reviewed journals such as Science and Engineering Ethics, Engineering Studies, History of Education, and Technology in Society. 


\section{Global Engineering Competency: Assessment Tools and Training Strategies}

\section{Introduction}

As many colleges and universities continue to expand and refine global learning opportunities for engineering students they face a complex array of challenges [1]. As one among many such concerns, administrators and other stakeholders increasingly expect that costly investments in international programming generate substantial benefits and impacts, including but not limited to growing the number of students served. Further, many programs are being asked to produce hard evidence of how research, work, study, and service abroad can enhance student learning and growth, synergistic with rising accountability pressures across the higher education landscape.

Fortunately, dozens of assessment instruments are available to measure many different facets of inter/cross-cultural competence, global competence, and related constructs [2]. Some tools, such as the Intercultural Development Inventory (IDI), are also synergistic with training programs designed to promote student learning and development [3]. However, debates persist about what tools are most valid and useful for assessing different kinds of interventions. Further, there remains a lack of high quality, validated assessment tools that are specifically focused on global engineering practice. As a consequence, administrators and researchers alike often find it difficult to collect robust, convincing evidence of how programs are supporting student growth and learning. There also remain unanswered questions about what program formats and training interventions have the largest measurable impacts on participants.

This paper responds to these challenges and pressures by introducing two tools that can be used for assessment and instructional purposes. We particularly emphasize the Global Engineering Competency - Situational Judgment Test (GEC-SJT) as a behavioral measure of competency. We more specifically present and discuss one sample assessment question drawn from a larger collection of scenarios focused on engineering in the Chinese national/cultural context. First, we review some relevant literature and background information about our larger research project. Second, we explain how the sample scenario was initially developed, as well as how we created a scoring key and collected validity evidence for the scenario through multiple rounds of data collection with subject matter experts (SMEs, i.e., practicing engineers). Third, we discuss how this scenario can be used for assessment purposes in the context of global engineering programs. Fourth, we present an instructional guide for those who might wish to use this type of scenario for training. Fifth and finally, we provide information about a complementary assessment tool we developed, the self-report Global Engineering Competency Scale (GECS).

This paper is expected to be of interest to faculty, staff, and administrators who are interested in assessing global engineering programs, as well as researchers who wish to measure global learning among engineering students, practicing professionals, and other populations. We also expect that instructors will benefit from this paper's discussion of scenario-based instruction as an accessible and impactful way to promote global competency and other professional learning outcomes among students in engineering and other professional fields. This work may especially resonate with those who are eager to help current and future engineers appreciate - and more effectively navigate - the kinds of cross-cultural dynamics often faced in global technical work. 


\section{Literature Review}

\section{Assessment Tools}

The extant literature reflects two prominent approaches to conceptualizing and assessing inter/cross-cultural competence and related constructs. First, so-called "compositional models" take a multidimensional approach to theorizing and measuring competence, often framed in terms of knowledge, skills, and attitudes (KSA) or cognitive, affective, and behavioral (CAB) dimensions [4]. Consistent with such models, many studies measure various individual characteristics and attributes proposed as foundational for inter/cross-cultural competence, often using self-rating scales [4-5]. In the engineering education field, studies taking this approach have used survey instruments like the Cultural Intelligence Scale (CQS) [6] and Miville-Guzman Universality-Diversity Scale [7-9].

The "developmental paradigm" or "developmental models" approach, by contrast, proposes that individuals can be placed on a continuum of intercultural competence levels or stages $[4,10]$. Assessment tools like the aforementioned IDI, based on Bennett's Developmental Model of Intercultural Sensitivity (DMIS) [11], can be used to evaluate where an individual falls on a developmental continuum [3]. The IDI has been used widely for research, training, and evaluation purposes in the international education field, including in large-scale national studies like the Georgetown Consortium Project [12] and multiple engineering education studies [8-9].

Another class of "hybrid" instruments purport to combine the compositional and developmental approaches. For instance, the Global Perspectives Inventory (GPI) is comprised of six scales that cover three dimensions: cognitive, intrapersonal, and interpersonal, i.e., "thinking, being, and relating" [13-14]. These scales and dimensions reflect influences from intercultural communication theory, which tends toward a compositional approach, as well as theories of intercultural maturity that take a more developmental view. GPI has been employed in large national studies of university students [14], and of engineering students more specifically [15].

Many of the assessment instruments mentioned above are backed by considerable evidence of their validity and reliability. Nonetheless, two potential shortcomings remain. First, none of these tools are contextualized or grounded in the realm of engineering practice. In light of this issue, other scholars have taken a compositional approach to creating new assessment tools that can be used to measure attributes or competencies viewed as especially relevant or important for global engineers. For example, the Engineering Global Preparedness Index (EGPI) is a 30-item survey organized around four subscales: global engineering ethics, global engineering efficacy, engineering global-centrism, and engineering community connectedness [16]. Other notable examples include the self-report items created by Mohtar and Dare [17] and Ball et al. [18].

Yet these instruments in turn reveal another possible shortcoming, namely their use of self-rating or self-reporting approaches to measuring competence. That is, all of these tools ask individuals to rate their own levels of competence, which presumes that the respondents: 1) understand the survey items as intended by the instrument developers, and 2) can and will perform an accurate and candid evaluation of their own levels of competence. While there is a long history of debate about these types of measurement challenges [19], in our own work we follow a complimentary 
line of scholarship involving the use of behavior-based assessments using a situational judgment test (SJT) format. This approach has the advantage of measuring the desired outcome or a proxy for the desired outcome (i.e., interculturally effective behaviors) that in turn likely reflects a variety of relevant competencies and attributes that are foundational for performance. While direct observations and third-party evaluations of competency are even stronger forms of evidence, the SJT format offers a behavior-based measure that is much easier and more scalable to administer. SJT measures can also be deployed in tandem with self-rating measures to extend the measurement space and/or triangulate across related measures. And as we describe in more detail below, scenario-based and situational assessment items are readily adaptable for use in instructional settings, e.g., courses or workshops focused on global engineering competency.

\section{Training Strategies}

Another topic of relevance for this paper centers on instructional strategies associated with both international education in general and global engineering education in particular. While a detailed discussion of these topics is beyond this paper's scope, here we note some highlights. First, there is a long and rich body of literature on intercultural education and training, including partially unique traditions and approaches in different fields and disciplines [20, Ch. 15-23]. A number of papers and reports have also surveyed and described approaches to developing global competency and related outcomes among engineering students and professionals [21-24].

While the extant literature on global engineering education has placed considerable emphasis on learning experiences that involve international travel (e.g., research, work, service, and study abroad), authors such as Downey et al. describe the "integrated class experience" as a "an athome effort to initiate students on the path to global competency in ways that fit their standard curricula" [21, p. 112]. Nonetheless, Grandin \& Hirleman note widespread consensus about the difficulty of "integrating global education into the engineering curriculum to impact all students" $[1$, p. 23], in no small part due to the "curricular rigidity" of most engineering degree programs $[1$, p. 11]. The present work responds to this challenge by describing a training approach that involves the use of short scenario-based exercises that can easily be folded into existing coursework. This technique has some semblance to Davis' "micro-insertion" approach to ethics pedagogy in science and engineering courses [25]. Our scenario-based approach also resonates with case-based approaches to engineering education [26]. Below we offer further discussion of some specific pedagogical strategies relevant to scenario-based prompts.

\section{Project Background}

In this section we provide background information to further contextualize this paper, as it discusses select facets of a larger research project. To begin, it is worth noting two main objectives for the parent project, namely to: 1) Generate an empirically-based definition and developmental theory of global engineering competency, and 2) Create and disseminate a valid and reliable situational judgment test (SJT) assessing 3-4 critical dimensions of global engineering competency. As described elsewhere, we approached the first objective by compiling a large collection of relevant literature (e.g., case studies and other characterizations of global technical work), and carrying out individual and group interviews with 25 experts, all of whom had previous and/or current job roles involving global technical work, typically in large 
corporations [27]. Analyzing this corpus of data and materials enabled us to in turn identify three more specific dimensions of global engineering competency (GEC): cross-cultural technical coordination; understanding and negotiating engineering cultures; and navigating ethics, standards, and regulations. During this part of the project we also narrowed our efforts to focus on six specific national/cultural contexts: China, France, Germany, India, Japan, and Mexico. These countries were selected in light of their status as rising or leading world economies, recognized influence in engineering and technology, and intensity of relations with the U.S.

Aligned with the second project objective, we next created a large collection of potential SJT assessment items covering the three GEC dimensions and six national/cultural contexts listed above. While described in more detail elsewhere [28], this process followed accepted best practices for SJT development and involved the following key steps: 1) draft at least 3 SJT item stems (i.e., paragraph-length descriptions of work situations or dilemmas that might be encountered by global engineers) for every possible combination of GEC dimension and country, and 2) create 5-9 response options (i.e., ways of handling a given situation with wide variations in likely effectiveness) for every item stem. We ultimately created 66 unique item stems, or 74 in total given that some were reused for use in multiple national/cultural contexts. Some item stems were also dropped due to difficulties writing suitable response options, resulting in a final set of 68 complete SJT items (i.e., stem and responses) ready for possible validation and deployment.

We additionally recognized that it would be beneficial to deploy a self-rating measure of global engineering competency as part of our initial efforts to validate the SJT items. This also led us to create and pilot a Global Engineering Competency Scale (GECS), as further described below.

\section{Development and Use of Sample GEC-SJT Assessment Item}

Due to the large number of complete SJT items that were originally created for this project, subsequent efforts have narrowed to focus on specific national/cultural contexts. This paper highlights one of our China assessment items. We initially focused on the Chinese context due to the large number of SJT items we created for China (18 total item stems, 16 fully developed), high levels of interest in China given its rising global prominence and intensity of its global interactions, and recognition of large cultural differences between China and the West. To further describe the SJT items we developed and how they might be used, we turn to the example item stem shown in Figure 1. In terms of GEC dimensions, this scenario falls in the area of "technical coordination" since it involves "getting things done" in the workplace amidst cultural differences, but with technical issues or expertise as a backdrop or contextual consideration. We decided to highlight a scenario from this category given a growing body of empirical evidence showing that technical coordination is a prominent aspect of engineering practice [29].

This and other similar scenarios are readily deployable in instructional settings as a discussion prompt, or used as an assessment tool with open-ended responses coded or scored using a standardized rubric. Indeed, such open-ended scenarios have seen some use for research and assessment in engineering education, both in general and in the area of global engineering education [31-32]. Nonetheless, using such scenarios for assessment poses a number of challenges, not least related to the time consuming task of developing and applying codebooks 
and/or scoring rubrics to evaluate the resulting data. Building on the item stem to create an SJT question opens up new assessment possibilities that are potentially much more scalable.

You are a newly appointed program manager in a global agricultural equipment company. To prepare for an upcoming training initiative, you have been asked to identify the top engineering talent at each international site. For your firm's operation in China, you engage your close colleague Wei Wang, who as plant manager supervises eight manufacturing engineers. When you explained the task to Wei during a recent teleconference, he said he understood it. You then asked him to e-mail you his numerical ratings for each of his engineer's technical abilities, timeliness in project work, and effectiveness on global teams. Yet when you receive his e-mail, you find that he has rated everyone essentially the same. What would you do?

Figure 1. Sample SJT Item Stem, GEC Scenario \#5: Rating for Training, Reproduced under Creative Commons License Attribution-NonCommercial-NoDerivatives 4.0 International [30]

For this particular scenario, we initially created seven possible response options with wide variations in probable effectiveness. Development of the response options was informed by our own understandings of Chinese culture and intercultural sensitivity, as well as evidence from other case studies and SMEs about how similar kinds of cases and situations are typically handled (or mishandled). By effectiveness, we mean that a given course of action would likely get the stated issue or problem resolved in a culturally appropriate manner. We next presented sets of 5-6 China SJT scenarios to SMEs $(n=14)$, all non-Chinese technical professionals with at least 1-3 years of experience working in China and/or with Chinese colleagues, vendors, clients, contractors, etc. For each SJT item presented, respondents were asked to indicate the plausibility of the item stem, as well as the plausibility and relative effectiveness of each associated response option on a scale ranging from 1 ("not at all effective") to 10 ("very effective"). We performed some basic statistical analyses with the goal of identifying at least 4-5 response options with relatively high levels of consensus among the SME effectiveness ratings, and trying to retain a mix of responses deemed both effective and not effective. If these conditions were not satisfied, we dropped the item stem. Table 1, below, presents the final set of five response options kept for this scenario, as well as the associated descriptive statistics.

Table 1. Response Options and Descriptive Statistics for SME Ratings, Scenario ID \#5 ( $\mathrm{n}=14$ )

\begin{tabular}{|l|c|c|c|}
\hline $\begin{array}{l}\text { Response Option } \\
\text { (Please rate the effectiveness of each item below on a scale } \\
\text { from 1 = Not at all effective to 10 = Very effective) }\end{array}$ & $\begin{array}{c}\text { SME } \\
\text { Mean } \\
(1-10)^{*}\end{array}$ & $\begin{array}{c}\text { SME } \\
\text { Std } \\
\text { Dev* }^{*}\end{array}$ & Effective? \\
\hline $\begin{array}{l}\text { a. Ask Wei whether he knows what he is doing, and have } \\
\text { him explain the reasoning behind his actions. }\end{array}$ & 2.7 & 1.8 & No \\
\hline $\begin{array}{l}\text { b. Create a new evaluation form that requires Wei to rank- } \\
\text { order the engineers. }\end{array}$ & 6.4 & 2.5 & Yes \\
\hline $\begin{array}{l}\text { c. Ask Wei to also send you written, descriptive evaluations } \\
\text { of each engineer. }\end{array}$ & 4.9 & 2.4 & Somewhat \\
\hline $\begin{array}{l}\text { d. Have a one-on-one conversation with Wei so you can go } \\
\text { through the ratings together and hear what he has to say } \\
\text { about each engineer. }\end{array}$ & 8.1 & 1.4 & Yes \\
\hline e. Randomly select one of the engineers for the training. & 1.9 & 2.4 & No \\
\hline
\end{tabular}

${ }^{*}$ Based on responses from $n=14$ subject matter experts (SMEs) 
In a parallel paper that is still under preparation, we report on our initial validation efforts focused on a final set of six China SJT items, including the sample scenario presented here. In summary, this research suggests that performance on the SJT measure (i.e., ability to rank the effectiveness of response options) was positively correlated with a Chinese cultural knowledge measure based on survey data collected from a large, random sample of practicing engineers $(n=400)$. This result provides important initial validity evidence for the measure. A multiple regression analysis also demonstrated that SJT performance was significantly predicted by Chinese cultural knowledge, total years of work experience, and a facet of personality called Agreeableness. These results therefore suggest that these six SJT items have considerable promise as a culture-specific approach to assessing global engineering competency.

To deploy such SJT items for assessment purposes, two general strategies are recommended. The first involves using the 1-10 rating scale given above to collect data, followed by use of a mean squared deviation (MSD) approach that involves: 1) calculating the difference between the participant's response and the SME rating for each item, 2) squaring this difference, 3) take the mean of these differences across all SJT items, and 4) multiply by -1 so that higher scores (i.e., those closer to zero) represent SJT ratings that are more similar to the SME ratings. Our research team is also working to identify scoring ranges so that an individual respondent's performance can be compared (e.g., in terms of percentile rank) to the aforementioned sample of 400 SMEs.

A simpler approach to assessment can also be built around the "Effective?" column in Table 1. For example, a respondent could be asked "Which of these actions (a-e) would you MOST (and/or LEAST) likely take?" or "Which of these actions (a-e) do you think is MOST (and/or LEAST) effective?" Alternately, each response option could be paired with three possible choices: "Not at all effective," "Somewhat Effective", or "Very effective." In either case, a given set of responses could be scored against the key presented in Table 1, with higher scores indicating that a respondent is better able to identify the relative effectiveness of each option. Those wishing to collect qualitative data for assessment or research purposes can also pair the scaled response items with open-ended prompts such as "Why would you take (or NOT take) this action? Please briefly explain." or "Please briefly explain the rationale for your effectiveness rating." As the following section notes, similar kinds of reflection or discussion prompts can also be useful when these same types of scenarios are deployed for training purposes.

\section{Instructional Guide for Sample GEC-SJT Assessment Item}

The instructional guide presented here has been developed so the sample SJT question presented above (Figure 1) can more easily be deployed by instructors in courses, workshops, and similar interventions. The guide was developed based in part on input from Qin Zhu, our Chinese coauthor, collaborator, and expert on Confucian philosophy and culture. We also drew insights from Hofstede's Cultural Dimensions theory [33]. As noted above, further evidence regarding the effectiveness of each response option through rating data collected from SMEs $(n=14)$ who were not Chinese but had worked extensively in Chinese contexts and/or with Chinese contacts.

Interpretation of Scenario Based on Culture and Context 
In Chinese culture, the concept of an individual person is historically very different from American and other Western conceptions [34]. Rather than seeing persons as largely autonomous and individualistic, Chinese culture has long tended to see a person primarily or even exclusively in terms of how s/he relates to other people in her/his network. For example, our colleague Wei Wang in the preceding scenario is not simply the individual person Wei Wang. Instead, Wei is a plant manager who supervises eight engineers, he is his parent's son, he is your colleague, etc.

In line with this conception, Wei likely gives a great deal of consideration regarding his relationships with his employees. Focused on interpersonal relationships, it is likely that Wei will be very hesitant to say anything negative about the engineers he supervises, as doing so could potentially have detrimental effects on their lives and careers. Furthermore, writing down his opinions and ratings of the engineers in an e-mail could make him uncomfortable, since written documentation is permanent. In fact, Wei would likely worry that his negative comments could one day come back to hurt him or his engineers.

This emphasis on relationships can further be understood from the viewpoint of Hofstede's Cultural Dimensions [35]. More specifically, on a scale of $0-100$, China scores 80 points on the "Power Distance" dimension. This score points to the fact that the Chinese people are typically more accepting of social hierarchy, including polarized relationships between leaders and followers, and recognizing the importance of respecting formal authority. Further, a low score of 20 points on the "Individualism" dimension reflects a strong sense of interdependence associated with living in a collectivist culture. Hence, most people in Chinese society have a self-image defined by "we" rather than "I." By contrast, the U.S. scores 40 on Power Distance, suggesting considerably more discomfort with social hierarchy, and a striking 91 on Individualism.

Two additional points are worth noting. First, the Chinese often rely heavily on intuition and then rationalize these intuitions. In the given scenario, Wei likely has an intuitive sense that it is wrong to numerically rate his employees against one another, potentially hurting their reputations, so he rationalizes that he should rate them all the same. And second, the Chinese often prefer to have conversations about sensitive material rather than writing something down. Since the written format can provide permanent evidence of an application of judgment, Wei likely prefers not to have formal documentation recorded in a situation where he is passing judgment on his colleagues.

As the preceding points suggest, someone not acquainted with these kinds of cultural differences between the China and U.S. (or West more generally) may indeed have difficulty understanding why Wei did not fulfill the original request as expected, much less know how to effectively and appropriately address the situation. The next section of the guide offers a more detailed discussion of the relative effectiveness of the five response options associated with this scenario.

\section{Interpretation of the Response Options Based on Culture and Context}

a. Ask Wei whether he knows what he is doing, and have him explain the reasoning behind his actions. 
(EFFECTIVENESS: LOW) This approach is likely ineffective because Wei probably fully understood the request, but does not believe it is culturally appropriate to judge his subordinates in the manner requested. By asking Wei to explain himself, you are showing a lack of awareness and sensitivity regarding Chinese cultural norms that are likely central to Wei's behavior.

b. Create a new evaluation form that requires Wei to rank-order the engineers.

(EFFECTIVENESS: MODERATE-HIGH) This approach would probably get you the results you are looking for (i.e., a ranked list of the Chinese employees), but it would likely still demonstrate a lack of awareness of Chinese cultural norms. This approach would still make Wei uncomfortable since it requires him to write down his judgments of the engineers, and those judgments would be forever immortalized because they were formally documented.

c. Ask Wei to also send you written, descriptive evaluations of each engineer.

(EFFECTIVENESS: MODERATE) This approach would likely provide you with detailed descriptions of each employee, but it might still be difficult for you to determine which employee to select based on this information. On the one hand, Wei would prefer to write down descriptive evaluations of each engineer as opposed to ranking them because he would have the opportunity to describe each person in context. On the other hand, Wei would probably write very similar or general things about each colleague, making it difficult to determine how they compare. Additionally, this approach requires Wei to write down evaluations with his name attached, which again would likely make him uncomfortable.

d. Have a one-on-one conversation with Wei so you can go through the ratings together and hear what he has to say about each engineer.

(EFFECTIVENESS: HIGH) This approach is likely the most effective and appropriate because it acknowledges a number of Chinese cultural preferences while allowing you to get the information you desire. By having a one-on-one conversation, Wei does not need to write down his ratings and opinions, thus making him more comfortable. Additionally, Wei will be able to describe his colleagues in context, which is very important in Chinese culture. The best way to understand what Wei thinks about the engineers is to ask him to provide specific examples of each engineer's skills. However, it would not be appropriate to record the conversation, as having concrete evidence about Wei's evaluation of each engineer would likely not be welcomed.

e. Randomly select one of the engineers for the training.

(EFFECTIVENESS: LOW) While this approach would result in selection of an engineer for the training, it is ineffective regardless of context. Someone who is very unfamiliar with Chinese culture might believe Wei was honest when he rated all the engineers the same, and believe they are all equal. This belief would justify simply choosing one, since they are all essentially the same. But someone who knows Chinese culture would be aware that the issue 
is more likely related to the fact that Wei did not want to provide written evidence of his opinions about the engineers.

Readers who are interested in more scenarios of this type can find another sample China scenario featured in a prior publication [28], and an adaptation of that scenario in the form of a short video vignette on YouTube [36] and an accompanying instructor's guide [37]. The video format is very engaging in training sessions, especially for student participants. Finally, the researchers have posted a large collection of other sample scenarios at https://geec.info/gec-about [38].

\section{Pedagogical Strategies}

We have found that scenarios of this type, whether used with or without the associated response options, have a number of advantages when deployed in instructional settings. First, they are relatively brief, making it easy to cover them in as little as 10-15 minutes in a course or workshop. Second, they tend to be very interactive and generate lively discussion, particularly when participants are asked to justify and defend their preferences for certain response options. On a related note, these discussions are often very generative, causing participants to identify alternate courses of action that combine or improve on the given, pre-defined choices. Third and finally, the scenarios are solidly contextualized in global engineering practice, thereby helping participants better identify with, and place themselves in the position of, the given protagonist.

Nonetheless, there remains the question of how one might go about facilitating classroom activities that involve such scenarios prompts. While many specific training strategies can be found in the aforementioned literature on international education and case-based learning, a few key points are worth noting. First, it is important to provide students with opportunities to develop and reflect on their own levels of cultural knowledge and intercultural sensitivity, as our own research and many other studies have frequently identified these characteristics as strongly predictive of one's ability to effectively and appropriately navigate cross-cultural situations.

Second and more practically, we have found it very beneficial to take a structured approach to scaffolding scenario-based training activities. For example, Describe-Interpret-Evaluate (DIE) and other similar frameworks have a long history of use in the international education field [39]. Rolfe, Freshwater, \& Jasper's reflective model offers a nice alternate set of three simple prompts: What? So what? Now what? [40]. As nicely summarized by Williams, the goal of such exercises is to "help people distinguish between objective descriptions, subjective interpretations, and emotional evaluations" [41, p. 149]. Further, encouraging participants to more systematically think through and reflect on a posed scenario and possible responses can help them identify gaps in their own knowledge, avoid making erroneous assumptions (e.g., such as those that are based on cultural stereotypes), and prevent jumping to conclusions prematurely.

Our own brief summary of the DIE phases is presented in Figure 2. To better orient participants to this method, it is often helpful to initially practice the approach using an ambiguous object, image, video clip, etc., as suggested by Bennett, Bennett, \& Stillings [42]. When a short scenario-based prompt is next introduced, it is often sufficient to simply begin discussion with the interpret phase since the description of the situation is brief and often speaks for itself. 
However, the describe phase is a very appropriate and important place to begin for a longer case study or a video vignette, especially to make sure participants factually grasp a given scenario.

\begin{tabular}{|c|c|}
\hline DIE Phase & Sample Discussion Prompts \\
\hline $\begin{array}{l}\text { Describe } \\
\text { (What?) }\end{array}$ & $\begin{array}{l}\text { - Factually, what is happening in this situation? } \\
\text { - In a few sentences, can you restate what is going on? } \\
\text { - What do we know about the various people involved? }\end{array}$ \\
\hline $\begin{array}{l}\text { Interpret or } \\
\text { Analyze } \\
\text { (So What?) }\end{array}$ & $\begin{array}{l}\text { - What is your interpretation of the given problem or dilemma? } \\
\text { - How would you feel if placed in this situation? } \\
\text { - What are some possible motives of the participants? } \\
\text { - What cultural considerations might be relevant in this situation? } \\
\text { - How might this situation look from other perspectives? }\end{array}$ \\
\hline $\begin{array}{l}\text { Evaluate } \\
\text { (Now What?) }\end{array}$ & $\begin{array}{l}\text { - What would you propose doing in this situation, and why? } \\
\text { - What are some possible pros and cons for each proposed course of action? } \\
\text { - How would you (and others) feel if a given course of action is taken? } \\
\text { - To what extent is a given response effective AND culturally appropriate? }\end{array}$ \\
\hline
\end{tabular}

Figure 2. DIE Phases and Sample Discussion Prompts

\section{Development and Use of Global Engineering Competency Scale (GECS)}

Complimentary to our work creating GEC-SJT items, we have also developed a culture-general, self-rating instrument called the Global Engineering Competency Scale (GECS). Here we offer an overview of the tool given its potential utility as another complimentary measure of global competency. As background, creation of the tool was inspired by a number of prior efforts to create and deploy self-rating measures focused on various dimensions and aspects and dimensions of global engineering $[17,18,21,32]$. More specifically, our research team adapted some items from these instruments and also wrote new items to create a pool of 28 items covering four main categories of interest: General Global Engineering Performance (6 items), Technical Coordination ( 7 items), Ethics, Standards, and Regulations (6 items), and Engineering Cultures (9 items). As this overview suggests, the latter three categories were inspired by the three dimensions of GEC we identified through our prior qualitative research efforts [27].

We next deployed all 28 items as part of the large-scale validation effort described above for the China GEC-SJT items, with the same sample of practicing engineers $(n=400)$ and using a 5-point Likert scale ranging from Strongly Disagree to Strongly Agree. This data was then randomly split into two sets of 200 responses to perform exploratory and confirmatory factor analyses. While the details of this process will be reported in more detail elsewhere, it is worth noting that this process allowed us to identify 12 specific items in the data that covered two distinct factors. The first, cognitive, includes 6 items that all start with phrases like "I am familiar with..." or "I understand...", e.g., "I am familiar with cross-national/cultural differences in engineering practice." The second factor is behavioral, and includes 6 items such as "I can practice engineering in an international setting." While the factor structure did not confirm to the GEC dimensions that we originally used to create and organize items, the types of factors that emerged are typical for a self-rating survey that uses a compositional approach to measure competence. 
The final set of 12 items comprising the Global Engineering Competency Scale (GECS) were included in the initial validation efforts associated with the aforementioned China GEC-SJT items. As we discuss in that body of work (manuscript under preparation), the two GECS factors did not predict performance on the SJT measure. In fact, scores on the GECS factors were negatively correlated with GEC-SJT performance at statistically significant levels, meaning that those who had higher self-reported GEC-SJT scores were actually less successful at rating the SJT response options. Interestingly, similar results were observed with another self-rating measure of cross/inter-cultural competency that was deployed in this study, namely the Cultural Intelligence Scale (CQS). Additionally, all four factors of CQS (cognitive, meta-cognitive, behavioral, and motivational) were significantly cross-correlated with both of the GECS factors.

While preliminary in nature, these results suggest some important implications. First, it may be the case that culture-general measures such as GECS and CQS are considerably distinct as compared to a culture-specific measure such as the China GEC-SJT items. That is, one's selfevaluation of one's own GEC or CQ in general may diverge considerably from one's ability to effectively span a specific national/cultural boundary. Second and potentially more concerning, the self-rating instruments may reflect some deeper measurement biases, including the tendency of less competent individuals to greatly overestimate their competency, or for some respondents to misinterpret survey items in ways that significantly skew the overall measure.

\section{Conclusion}

In this paper we introduce a sample GEC-SJT item and offer a detailed discussion of how it can be used for both assessment and instructional purposes. We hope that the information presented here will prove inspiring and useful for instructors, staff, and administrators who are involved with creating and running global engineering programs. In particular, we propose SJT items as a scalable, behavior-based approach to measuring global engineering competency. The SJT item stems and response options can also be adapted and deployed as engaging learning activities.

Nonetheless, what has been presented here is just a small slice of a much larger project. As noted above, the research team has created an online database of open-ended scenarios and SJT items that can be searched by country, GEC dimension, and many other relevant characteristics (e.g., industry sector, engineering discipline, types of boundaries spanned, etc.) [38]. The research team has also created a companion web site call the Global Assessment Platform (GAP), where interested individuals can get immediate access to the full set of six China scenarios and view their results in relation to the SME data mentioned in this paper, as well as similar sets of scenarios and scoring data focused on the national/cultural contexts of India and Germany [43]. Since future efforts to validate our SJT items will require large amounts of data, we hope to work with the larger community to more widely deploy our instruments for research, assessment, and instruction purposes. In doing so, we aim to support wider efforts to help current and future engineers learn to work more effectively and appropriately across cultural boundaries.

\section{Acknowledgments}

This material is based upon work supported by the National Science Foundation under Grant Nos. 1160455 and 1254323. Any opinions, findings, and conclusions or recommendations 
expressed in this material are those of the author(s) and do not necessarily reflect the views of the National Science Foundation. We also thank affiliates of Prof. Jesiek's Global Engineering Education Collaboratory (GEEC) and Prof. Woo's Laboratory for Understanding Careers and Individual Differences (LUCID) for their extensive assistance with developing SJT items.

\section{References}

[1] Grandin, J., \& Hirleman, E. D. (2009). Educating engineers as global citizens: A call for action / A report of the national summit meeting on the globalization of engineering education. Online Journal of Global Engineering Education, 4(1): 1-28.

[2] Fantini, A. E. (2009). Assessing intercultural competence: Issues and tools. In D. K. Deardorff (Ed.), The SAGE Handbook of Intercultural Competence (pp. 456-476). Thousand Oaks, CA: SAGE.

[3] Hammer, M. R. (2009). The intercultural development inventory: An approach for assessing and building intercultural competence. In M.A. Moodian (Ed.) Contemporary Leadership and Intercultural Competence (pp. 203-218). Thousand Oaks, CA: Sage.

[4] Spitzberg, B. H., \& Changnon, G. (2009). Conceptualizing intercultural competence. In D. K. Deardorff (Ed.), The SAGE Handbook of Intercultural Competence (pp. 2-52). Thousand Oaks, CA: SAGE.

[5] Fantini, A. E. (2009). Assessing intercultural competence: Issues and tools. In D. K. Deardorff (Ed.), The SAGE Handbook of Intercultural Competence (pp. 456-476). Thousand Oaks, CA: SAGE.

[6] Mazzurco, A., Jesiek, B. K., \& Ramane. K. (2012). Are engineering students culturally intelligent? Preliminary results from a multiple group study. Proceedings of the 2012 ASEE Annual Conference and Exposition, San Antonio, TX, June 10-13, 2012.

[7] Jesiek, B. K., Shen, Y., \& Haller, Y. (2012). Cross-cultural competence: A comparative assessment of engineering etudents. International Journal of Engineering Education, 28(1): 144-155.

[8] Bielefeldt, A., Paterson, K. \& Swan, C. (2010). Measuring the value added from service learning in project-based engineering education. International Journal of Engineering Education, 26(3): 535-546.

[9] Groll, L. (2013). Negotiating Cultural Humility: First-Year Engineering Students' Development in a Life-Long Journey (Unpublished doctoral dissertation). Purdue University, West Lafayette, IN.

[10] Hammer, M. (2012). The intercultural development inventory: A new frontier in assessment and development of intercultural competence. In M. Vande Berg, R. M. Paige, \& K. H. Lou (Eds.), Student Learning Abroad (Ch. 5, pp. 115-136). Sterling, VA: Stylus Publishing.

[11] Bennett, M. J. (2017). Developmental model of intercultural sensitivity. The International Encyclopedia of Intercultural Communication (pp. 1-10). John Wiley \& Sons, Inc.

[12] Vande Berg, M., Connor-Linton, J., \& Paige, R. M. (2009). The Georgetown consortium project: Interventions for student learning abroad. Frontiers: The Interdisciplinary Journal of Study Abroad, 18: 1-75. 
[13] Merrill, K. C., Braskamp, D. C., \& Braskamp, L. A. (2012). Assessing individuals' global perspective. Journal of College Student Development, 53(2): 356-360.

[14] Braskamp, L. A., Braskamp, D. C., Merrill, K. C., \& Engberg, M. (2013). Global Perspective Inventory (GPI): Its Purpose, Construction, Potential Uses, and Psychometric Characteristics. Chicago, IL: Global Perspective Institute, Inc. Retrieved from http://citeseerx.ist.psu.edu/viewdoc/download?doi=10.1.1.584.9216\&rep=rep1\&type=pdf

[15] Levonisova, S. V., Savage, R. E., Streiner, S. C., McCave, E. J., Ragusa, G., Matherly, C., Benson, L., Besterfield-Sacre, M., \& Shuman, L. J. (2015). Identifying factors that enhance undergraduate engineering students' global preparedness. Proceedings of the 2015 ASEE Annual Conference and Exposition, Seattle, WA, June 14-17, 2015.

[16] Ragusa, G. (2011). Engineering preparedness for global workforces: Curricular connections and experiential impacts. Proceedings of the 2011 ASEE Annual Conference and Exposition, Vancouver, BC, June 26-29, 2011.

[17] Mohtar, R. H., \& Dare, A. E. (2012). Global design team: A global service-learning experience. International Journal of Engineering Education, 28(1): 169-182.

[18] Ball, A. G., Zaugg, H., Davies, R., Tateishi, I., Parkinson, A. R., Gensen, C. G., \& Magleby, S. P. (2012). Identification and validation of a set of global competencies for engineering students. International Journal of Engineering Education, 28(1): 156-168.

[19] Chan, D. (2009). So why ask me? Are self-report data really that bad? In C. E. Vance \& R. J. Vandenberg, Statistical and Methodological Myths and Urban Legends: Doctrine, Verity and Fable in the Organizational and Social Sciences (pp. 309-336). New York and London: Routledge.

[20] Deardorff, D. K. (Ed.). (2009). The SAGE Handbook of Intercultural Competence. Thousand Oaks, CA: SAGE.

[21] Downey, G., Lucena, J. C., Moskal, B., Bigley, T., Hays, C., Jesiek, B., Kelly, L., Lehr, J., Miller, J., Nichols-Belo, A., Ruff, S., \& Parkhurst, R. (2006). The globally competent engineer: Working effectively with people who define problems differently. Journal of Engineering Education, 105(2): 107-122.

[22] Parkinson, A. (2007). Engineering study abroad programs: Formats, challenges, best practices. Online Journal of Global Engineering Education, 2(2).

[23] Johri, A., \& Jesiek, B. K. (2014). Global and international issues in engineering education. In A. Johri \& B. M. Olds (Eds.), Cambridge Handbook of Engineering Education Research (pp. 655-672). New York, NY: Cambridge University Press.

[24] Grandin, J., \& Hedderich, N. (2009). Intercultural competence in engineering: Global competence for engineers. In D. K. Deardorff (Ed.), The SAGE Handbook of Intercultural Competence (pp. 362-373). Thousand Oaks, CA: SAGE.

[25] Davis, M. (2006). Integrating ethics into technical courses: Micro-insertion. Science and Engineering Ethics, 12(4): 717-730.

[26] Richards, L. G., Gormon, M., Scherer, W. T., \& Landel, R. D. (1995). Promoting active learning with cases and instructional modules. Journal of Engineering Education, 84(4): 375-381.

[27] Jesiek, B. K., Zhu, Q., Woo, S. E., Thompson, J., \& Mazzurco, A. (2014). Global engineering competency in context: Situations and behaviors. Online Journal of Global Engineering Education, 8(1). 
[28] Jesiek, B. K., Woo, S. E., Zhu, Q., Ramane, K. D., \& Choudhary, N. (2015). Defining and assessing global engineering competency: Methodological reflections. Proceedings of the 2015 ASEE Annual Conference and Exposition, Seattle, WA, June 14-17, 2015.

[29] Trevelyan, J. (2007). Technical coordination in engineering practice. Journal of Engineering Education, 96(3), 191-204.

[30] Jesiek, B. K., \& Woo, S. E. (Eds.). (2018). GEC scenario \#5: Rating for training. Retrieved from https://geec.info/gec05

[31] Jesiek, B. K., \& Woo, S. E. (2011). Realistic assessment for realistic instruction: Situational assessment strategies for engineering education and practice. Proceedings of the SEFI Annual Conference 2011, Lisbon, Portugal, September 27-30, 2011.

[32] Jesiek, B. K., Haller, Y., \& Thompson, J. (2014). Developing globally competent engineering researchers: Outcomes-based instructional and assessment strategies from the IREE 2010 China research abroad program. Advances in Engineering Education, 4(1).

[33] Hofstede, G. (2011). Dimensionalizing cultures: The Hofstede model in context. Online Readings in Psychology and Culture, 2(1). https://doi.org/10.9707/2307-0919.1014

[34] Nisbett, R. E. (2004). The Geography of Thought: How Asians and Westerners Think Differently... and Why. New York, NY: Free Press.

[35] Hofstede Insights. (2018). Compare countries. Helsinki, Finland: Hofstede Insights. Retrieved from https://www.hofstede-insights.com/product/compare-countries/

[36] Jesiek, B. K. (Director and Producer) (2013). Global engineering competency vignette \#1 [Video file]. Retrieved from https://www.youtube.com/watch?v=Hbat8X96m9g

[37] Jesiek, B. K. (2014). Global engineering competency vignette \#1: Instructor's guide. Retrieved from http://geec.info/public/REE\%20GEC\%20Shanghai\%20Instructor's\%20Guide.pdf

[38] Jesiek, B. K., \& Woo, S. E. (Eds.). (2018). GEC Project - About. Retrieved from https://geec.info/gec-about

[39] Nam, K. A., \& Condon, J. (2010). The DIE is cast: The continuing evolution of intercultural communication's favorite classroom exercise. International Journal of Intercultural Relations, 34(2010): 81-87.

[40] Rolfe, G., Freshwater, D., \& Jasper, M. (2001) Critical Reflection in Nursing and the Helping Professions: A User's Guide. Basingstoke: Palgrave Macmillan.

[41] Williams, T. R. (2013). Examine your LENS: A tool for interpreting cultural differences. Frontiers: The Interdisciplinary Journal of Study Abroad, 22: 148-165.

[42] Bennett, J., Bennett, M., \& Stillings, K. (1977). Intercultural Communication Workshop Facilitator's Manual. Portland, OR: Intercultural Communication Institute. Retrieved from http://intercultural.org/training-and-assessment-tools.html\#DIE

[43] Global Engineering Education Collaboratory (GEEC). (2018). Global Assessment Platform (GAP). Retrieved from https://geec.info/gap/ 